\title{
Observation of Wet Samples Using a Novel Atmospheric Scanning Electron Microscope
}

\author{
Yusuke Ominami ${ }^{1}$, Shinsuke Kawanishi ${ }^{1}$, Tatsuo Ushiki ${ }^{2}$, Sukehiro Ito ${ }^{1}$ \\ 1. Hitachi High-Technologies Corporation, 882, Ichige, Hitachinka-shi, Ibaraki-ken, 312-8504, \\ Japan \\ 2. Niigata University Graduate School of Medical and Dental Sciences, 1-757 Asahimachi-dori, \\ Chuo-ku, Niigata-city, Niigata-ken, 951-9510, Japan
}

The scanning electron microscope (SEM) has been used as a powerful tool for providing surface information of micro and nanostructures. In recent years, SEM methods for observing wet samples under atmospheric pressure have been reported by some investigators [1-2]. With these methods, the sample space is separated by a thin transparent membrane from vacuum environment where electron beam is propagated, and samples attaching to the membrane are observed by SEM.

In the present study, we developed a table-top atmospheric SEM (ASEM) for observing samples which are present in ambient air conditions but are separated from the membrane (Fig.1). Our ASEM has an inner chamber inside a regular specimen chamber. This inner chamber is equipped with an attachable thin membrane on its roof. When a sample should be exchanged, the specimen stage is extracted from the inner chamber (Fig. 1(b)). In this configuration, the environment around the sample can be kept in ambient air conditions and changed by evaporation with an additional vacuum pump (Fig. 1(c)). Moreover, the higher vacuum observation can be also performed after removing the membrane from the inner chamber (Fig. 1(d)). This means that our ASEM enables observation of samples under not only atmospheric but also various-ranged vacuum pressures.

Using our ASEM, we observed wet regenerated cellulose fibers at atmospheric pressure (Fig. 2). After obtaining an image of wet fibers (Fig. 2(a)), the specimen chamber was evaporated using the additional vacuum pump and the same fibers were observed in different pressure conditions. Measurement from the SEM images shows that the fibers shrunk approximately $25 \%$ in diameter after evaporation (Fig. 2(b) and (c)), indicating that the fibers swell with water. We also succeeded in observing wet biological samples including blood cells and renal glomerulus at atmospheric pressure (Fig. 3(a) and (b)). We further compared an ASEM image of the mildew fungus with its light microscopic image, (Fig. 3(c) and (d)). No additional preparation of samples are not required between light microscope and our ASEM, which allows to be widely used for evaluation of wet materials observed using ASEM.

\section{References}

[1] S Thiberge, et al., Rev. of Scientific Instruments, 75, 2280, (2004)

[2] H. Nishiyama, et al., Microsc. Microanal., 15938 (2009) 

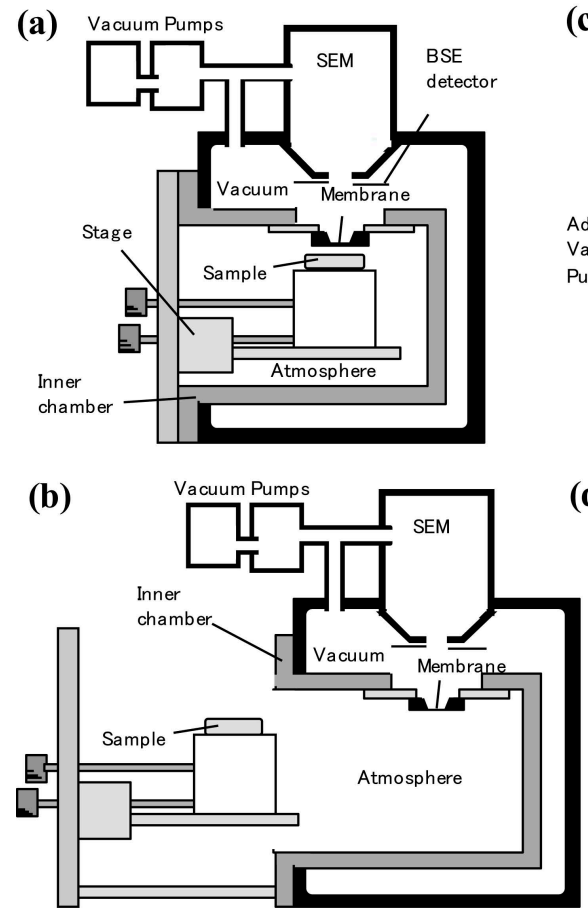

(c)

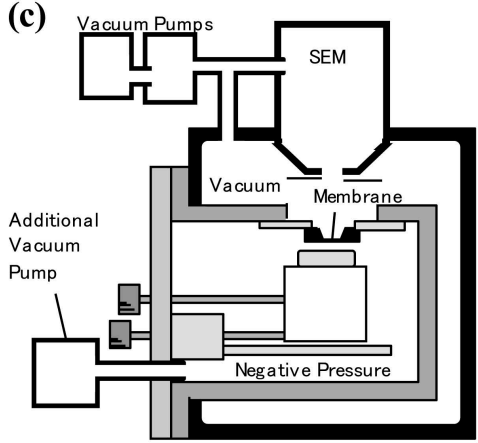

(d)

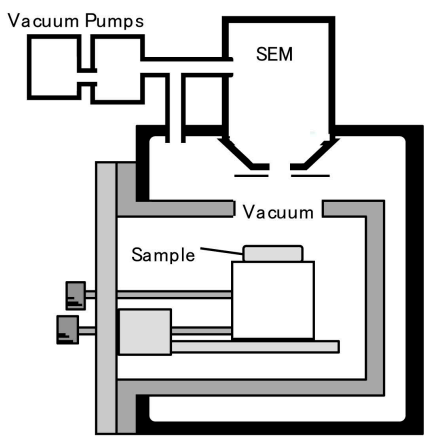

(e)

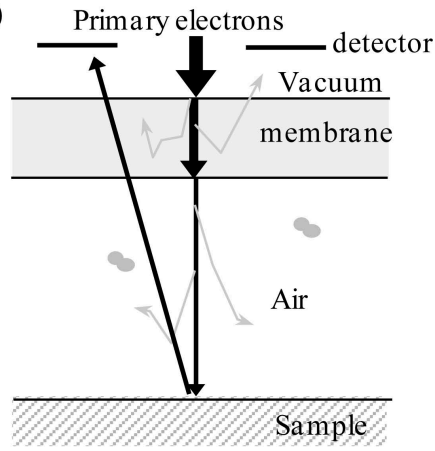

(f)

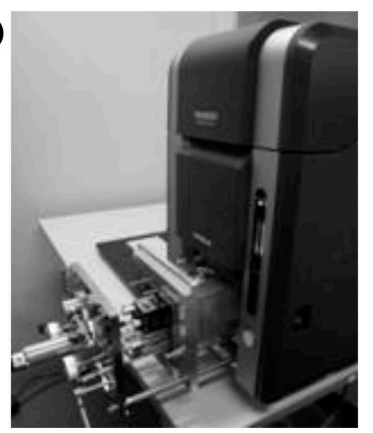

Fig. 1 (a)-(d) Schemes of the proposed table-top ASEM. (a) Imaging mode at (a) atmospheric pressure (10 $\mathrm{Pa}$ ), (c) negative pressure $\left(<10^{5} \mathrm{~Pa}\right)$, and (d) low vacuum pressure $\left(<10^{1} \mathrm{~Pa}\right)$. (b) Position of specimen-exchange. (e) Schematics of events of primary electrons. (e) Photograph of developed ASEM
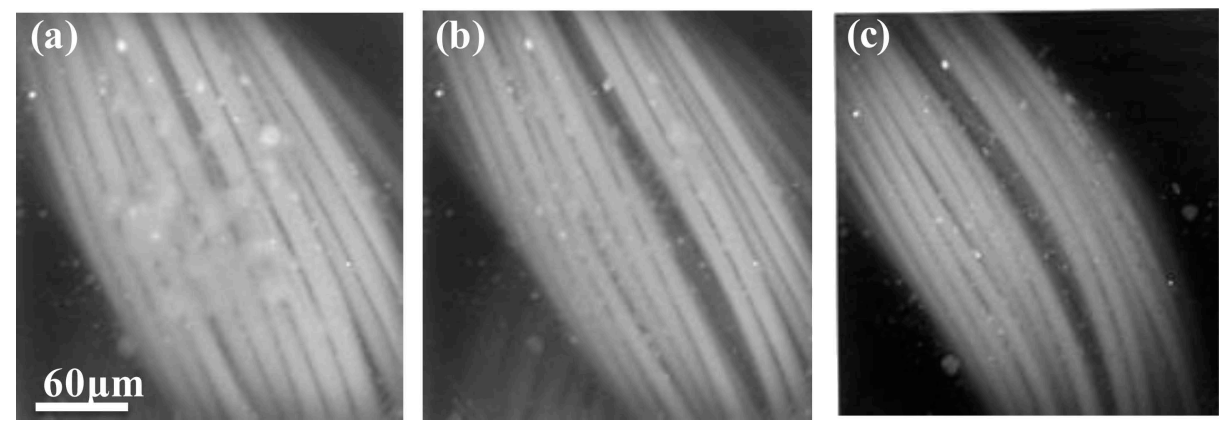

Fig. 2 SEM images of regenerated cellulose fibers in a drying process (from $100 \mathrm{kPa}$ to $10 \mathrm{kPa}$ ).
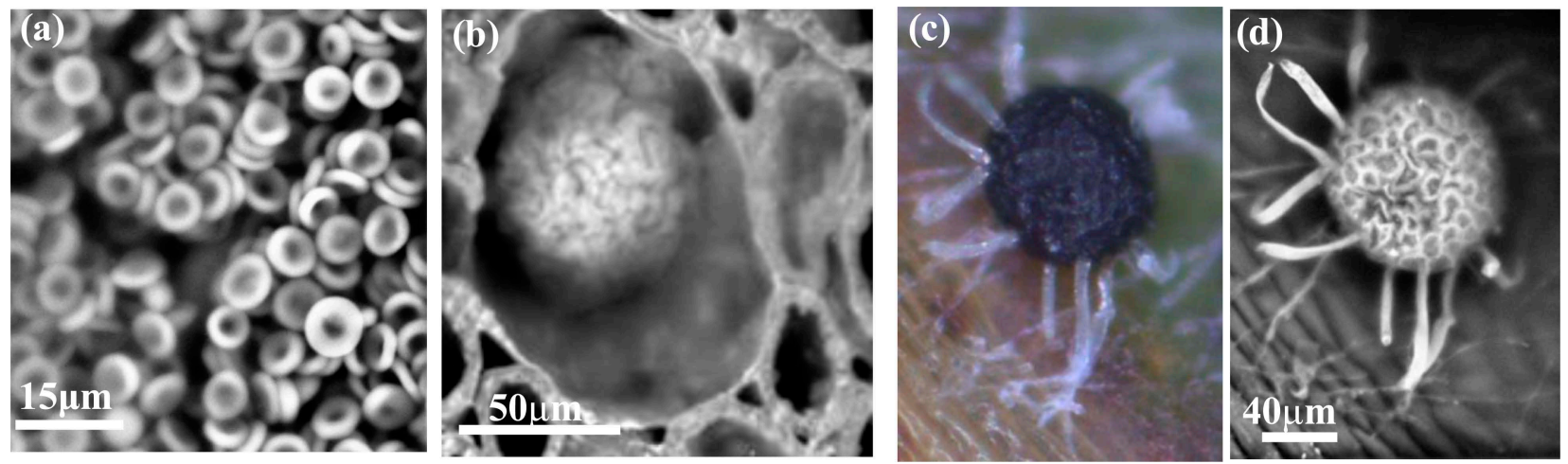

Fig. 3. SEM images taken at $10^{5} \mathrm{~Pa}$ (a) fixed blood cells (unstained), (b) Pt stained renal glomerulus, (c)(d) mildew fungus on a leaf observed using light microscope and our ASEM. 